\title{
Formulation of the MACE Filter as a Linear Associative Memory
}

\author{
John W. Fisher III Member, IEEE, Jose C. Principe Senior Member, IEEE
}

\begin{abstract}
We show that the minimum average correlation energy (MACE) filter can be formulated as a linear associative memory (LAM) trained with pre-whitened exemplars. Using this formulation, iterative methods which are numerically stable can be used to obtain the equivalent MACE filter coefficients in the space domain.
\end{abstract}

\section{INTRODUCTION}

The minimum average correlation energy (MACE) filter of [Mahal, Ravi], produces sharp correlation peaks over the recognition class in pattern recognition problems. It is known that the MACE filter of [Mahal, Ravi] can be formulated as a pre-whitening filter cascaded with a linear synthetic discriminant function. It will be shown that the MACE filter is equivalent to a linear associative memory (LAM) trained with pre-whitened patterns and as a result its coefficients can be computed iteratively via the LMS algorithm in the space domain. Experimental results will also be shown.

\section{MACE Filter Formulation}

The MACE filter minimizes the average output correlation energy of a filter. The optimization is subject to a set of linear peak constraints when the filter is centered on a pattern in the recognition class. The correlation function of the $i^{\text {th }}$ image with the filter $h\left(n_{1}, n_{2}\right)$ is denoted [Mahal]

$$
g_{i}\left(n_{1}, n_{2}\right)=x_{i}\left(n_{1}, n_{2}\right) \otimes \otimes h\left(n_{1}, n_{2}\right)
$$

where $\otimes \otimes$ indicates the 2-D correlation operator, and $h$ has a rectangular region of support which is $N_{1} \times N_{2}$. The DFT of the $i^{\text {th }}$ training image $x_{i}$ is denoted $X_{i}\left(k_{1}, k_{2}\right)$. The correlation plane energy

The authors are with the Computational Neuroengineering Laboratory, Department of Electrical Engineering, University of Florida, Gainesville, FL 32611

This work has been partially supported by NFS grant ECS \#920878. for the $i^{\text {th }}$ training image is [Mahal]

$$
\begin{aligned}
E_{i} & =\sum_{n_{1}} \sum_{n_{2}}\left|g_{i}\left(n_{1}, n_{2}\right)\right|^{2} \\
& =\frac{1}{N_{1} N_{2}} \sum_{k_{1}} \sum_{k_{2}}\left|G_{i}\left(k_{1}, k_{2}\right)\right|^{2} \\
& =\frac{1}{N_{1} N_{2}} \sum_{k_{1}} \sum_{k_{2}}\left|H\left(k_{1}, k_{2}\right)\right|^{2}\left|X_{i}\left(k_{1}, k_{2}\right)\right|^{2}
\end{aligned}
$$

The average correlation plane energy, $E$, over $N_{t}$ images is then

$$
E=\frac{1}{N_{t}} \sum_{i} E_{i}
$$

Reordering the images by row or column into column vectors, equation 1 can be written in a quadratic form as

$$
\begin{aligned}
E & =\frac{1}{N_{1} N_{2}}\left(\frac{1}{N_{t}} \sum_{i}\left(\mathbf{H}^{\dagger} \mathbf{D}_{i} \mathbf{H}\right)\right) \\
& =\frac{1}{N_{1} N_{2}}\left(\mathbf{H}^{\dagger} \mathbf{D H}\right),
\end{aligned}
$$

where $\mathbf{H}^{\dagger}$ is the hermitian transpose of the column vector $\mathbf{H}$, and $\mathbf{D}_{i}$ is a diagonal matrix whose diagonal elements are $\left|X_{i}\left(k_{1}, k_{2}\right)\right|^{2}$ ordered by row or column in the same manner as $H \rightarrow \mathbf{H}$. The minimization of the average correlation energy as defined above is a quadratic optimization problem. Imposing peak constraints when the filter is centered over a pattern in the recognition class is equivalent in the frequency domain to

$$
\mathbf{X}^{\dagger} \mathbf{H}=\mathbf{d}
$$

where columns of the matrix

$$
\mathbf{X}=\left[\mathbf{X}_{1} \cdots \mathbf{X}_{N_{t}}\right] \in \mathcal{Z}^{\left(N_{1} N_{2}\right) \times N_{t}}
$$

contain the reordered DFT coefficients of the training images and the column vector,

$$
\mathbf{d} \in \mathcal{Z}^{N_{t} \times 1}
$$


contains the corresponding constrained peak response values. Equation 3 is correct when the space domain and spectral domain coefficients are related by a unitary transformation as will be assumed here. If the DFT is defined as in [Opp and Schaf] then the right-hand side of the equation would need a factor of $N_{1} N_{2}$ and consequently the solution in the spectral domain will require the same factor. The solution for $\mathbf{H}$ can be found using the method of Lagrange multipliers and is given in [Mahal, Ravi] as

$$
\mathbf{H}=\mathbf{D}^{-1} \mathbf{X}\left(\mathbf{X}^{\dagger} \mathbf{D}^{-1} \mathbf{X}\right)^{-1} \mathbf{d}
$$

\section{Whitening/SDF INTERPRETATION}

If the matrix $\mathbf{D}^{\mathbf{- 1}}$ can be factored as $\mathbf{D}^{\mathbf{- 1}}=\mathbf{B}^{\dagger} \mathbf{B},{ }^{1}$ then equation 4 can be written as

$$
\mathbf{H}=\mathbf{B}^{\dagger} \mathbf{B} \mathbf{X}\left(\mathbf{X}^{\dagger} \mathbf{B}^{\dagger} \mathbf{B} \mathbf{X}\right)^{-1} \mathbf{d}
$$

or, by introducing a new variable $\mathbf{Y}=\mathbf{B X}$, equation 4 becomes

$$
\mathbf{H}=\mathbf{B}^{\dagger} \mathbf{Y}\left(\mathbf{Y}^{\dagger} \mathbf{Y}\right)^{-\mathbf{1}} \mathbf{d}
$$

which is equivalent to a whitening filter followed by a projection synthetic discriminant function (SDF) [Mahal]. The SDF portion of equation 6 can be computed in the space domain as

$$
\mathbf{h}^{\prime}=\mathbf{y}\left(\mathbf{y}^{\dagger} \mathbf{y}\right)^{-1} \mathbf{d},
$$

yielding in the spectral domain

$$
\mathbf{H}=\mathbf{B}^{\dagger} \mathbf{H}^{\prime} \text {. }
$$

\section{Linear Associative Memory Formulation}

The LAM is a linear transformation that maps a set of inputs to a corresponding set of outputs. A general form of the LAM is

$$
\mathbf{W}^{\dagger}(\mathbf{A x})=\mathbf{d}^{\dagger}
$$

where the columns of the matrix

$$
\mathbf{x}=\left[\mathbf{x}_{1} \ldots \mathbf{x}_{N_{t}}\right] \in \mathcal{Z}^{\left(N_{1} N_{2}\right) \times N_{t}}
$$

contain the reordered elements of the input patterns and the matrix

$$
\mathbf{A} \in \mathcal{Z}^{\left(N_{1} N_{2}\right) \times\left(N_{1} N_{2}\right)}
$$

${ }^{1}$ in the case of the MACE filter $D$, and hence $D^{-1}$ are diagonal matrices and this factorization is trivial. The are other filters formulated in a similar manner as the MACE filter in which $D$ is defined differently and is not necessarily diagonal. is any nonsingular linear transformation matrix ${ }^{2}$, and $\mathbf{d}$, the column vector of desired output responses, is equal to the peak constraint vector of the MACE filter.

The problem is to find a suitable vector $\mathbf{W}$, that satisfies the matrix equation 7 . For the underdetermined case, the minimum $L_{2}$-norm solution is the vector, $\mathbf{W}$, that minimizes the optimization criterion

$$
E=\mathbf{W}^{\dagger} \mathbf{W}
$$

subject to the linear constraints of equation 7 . As in the case of the MACE filter this solution can be found using Lagrange multipliers to minimize the augmented criterion

$$
E_{a}=E+\left(\mathbf{W}^{\dagger}(\mathbf{A x})-\mathbf{d}^{\dagger}\right) \lambda
$$

which at the minimum norm solution must satisfy

$$
\begin{gathered}
\frac{\partial E_{a}}{\partial \mathbf{W}}=2 \mathbf{W}+(\mathbf{A x}) \lambda=0 \quad \text {, or } \\
\mathbf{W}=-\frac{1}{2}(\mathbf{A x}) \lambda
\end{gathered}
$$

Substituting equation 8 into the LAM equation 7 and assuming the columns of $\mathbf{x}$ are linearly independent yields

$$
\lambda=-2\left(\mathbf{x}^{\dagger} \mathbf{A}^{\dagger} \mathbf{A} \mathbf{x}\right)^{-1} \mathbf{d}
$$

Substituting this result into equation 8 gives the final solution as

$$
\mathbf{W}=\mathbf{A} \mathbf{x}\left(\mathbf{x}^{\dagger} \mathbf{A}^{\dagger} \mathbf{A} \mathbf{x}\right)^{-1} \mathbf{d}
$$

This solution is in general agreement with that given in [Kohonen, p. 187] as

$$
\mathbf{W}=\left((\mathbf{A x})^{\dagger}\right)^{\dagger} \mathbf{d}
$$

where $\left((\mathbf{A x})^{\dagger}\right)^{\dagger}$ is Moore-Penrose (pseudo) inverse of $(\mathbf{A x})^{\dagger}$.

Equation 7 can be viewed as a linear pre-processor followed by a linear associative memory. The matrix, $\mathbf{h}$, incorporating both the pre-processing and linear associative memory such that $\mathbf{h}^{\dagger} \mathbf{x}=\mathrm{d}^{\dagger}$ is

$$
\mathbf{h}=\mathbf{A}^{\dagger} \mathbf{A} \mathbf{x}\left(\mathbf{x}^{\dagger} \mathbf{A}^{\dagger} \mathbf{A} \mathbf{x}\right)^{-1} \mathrm{~d} .
$$

The similarities of equations 5 and 11 are obvious, the significant difference being that the transformation of equation 11 operates on the input patterns in

\footnotetext{
${ }^{2}$ Typically $A$ is the identity matrix, but this generalization will be useful later.
} 
the space domain, while the transformation of equation 5 operates in the input patterns in the spectral domain. Note that there is a unitary transformation matrix $\Phi$, such that

$$
\Phi \mathbf{x}=\mathbf{X}, \mathbf{X}=\Phi^{\dagger} \mathbf{x}, \text { and } \Phi \Phi^{\dagger}=\Phi^{\dagger} \Phi=I,
$$

whose elements are dependent on the reordering of the 2-D patterns in both the space and spectral domains. Upon setting $\mathbf{A}=\mathbf{B} \Phi$ equation 11 becomes

$$
\mathbf{h}=\Phi^{\dagger} \mathbf{B}^{\dagger} \mathbf{B X}\left(\mathbf{X}^{\dagger} \mathbf{B}^{\dagger} \mathbf{B X}\right)^{-1} \mathbf{d}
$$

Transforming equation 12 to the spectral domain via $\mathbf{H}=\Phi \mathbf{h}$ yields

$$
\mathbf{H}=\mathbf{B}^{\dagger} \mathbf{B X}\left(\mathbf{X}^{\dagger} \mathbf{B}^{\dagger} \mathbf{B} \mathbf{X}\right)^{-1} \mathbf{d},
$$

which is equivalent to the MACE filter solution of equation 5 .

As stated in [Mahal] any nonsingular D, not necessarily the average power spectrum, may be used for the optimization criterion in which case the above equivalency will still hold under the additional condition that $\mathbf{D}^{-\mathbf{1}}$ can be decomposed as $\mathbf{D}^{-1}=\mathbf{B}^{\dagger} \mathbf{B}$.

\section{Iterative Computation of MACE Filter CoEFFicients}

One advantage of the linear associative memory formulation is that the filter can be computed iteratively using a gradient procedure such as the WidrowHoff (LMS) rule

$$
\mathbf{W}_{\mathbf{k}+\mathbf{1}}^{\dagger}=\mathbf{W}_{\mathbf{k}}^{\dagger}+\mu\left(\mathbf{d}^{\dagger}-\mathbf{W}_{\mathbf{k}}^{\dagger}(\mathbf{A x})\right)(\mathbf{A x})^{\dagger}
$$

Conditions for stability of this algorithm via proper choice of the scalar $\mu$ are described in [Widrow, pp. 99-115]. This eliminates the necessity for computing the inverse of the matrix ( $\mathbf{x}^{\mathrm{T}} \mathbf{A}^{\mathrm{T}} \mathbf{A x}$ ), which may be singular in practical situations, and the need for working with large matrices[Duda, p. 156]. Another advantage of the LMS rule is that it only requires local information. If the input training patterns are adequately pre-whitened the LMS rule will be equivalent to Newton's method which will result in a significantly faster convergence rate.

The LMS rule only modifies the component of the projection vector in the range of the matrix $(\mathbf{A x})^{\mathbf{T}}$, therefore initializing the projection vector to zero will insure convergence to the minimum norm solution of equation 9 [Amit, p. 105].

\section{Vi. Experimental Results}

Figure 1 shows the image used for experimentation. Three balls were dropped in front of a high speed video camera and consecutive frames were digitized.
The numbered boxes in the figure show the $24 \times 24$ pixel regions used for training patterns. The regions are labeled 0 through 6 , with the first three containing the images of the balls.

The purpose of these experiments was to illustrate the equivalence of the MACE filter to a LAM trained with patterns pre-processed by the appropriate linear transformation.

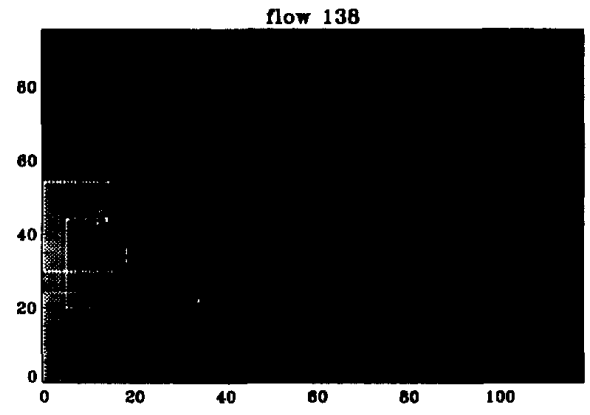

Figure 1: Image of balls used for experiment, The numbered boxes show the regions of the image used for training patterns.

\section{A. Case I}

In the first experiment only regions 0 through 2 were used as training patterns. The whitening filter was computed using the average spectrum of these input patterns. The MACE filter coefficients were computed using equation 4 . The LAM coefficients were computed iteratively using the LMS (Widrow-Hoff) rule and training with pre-whitened patterns in the space domain.

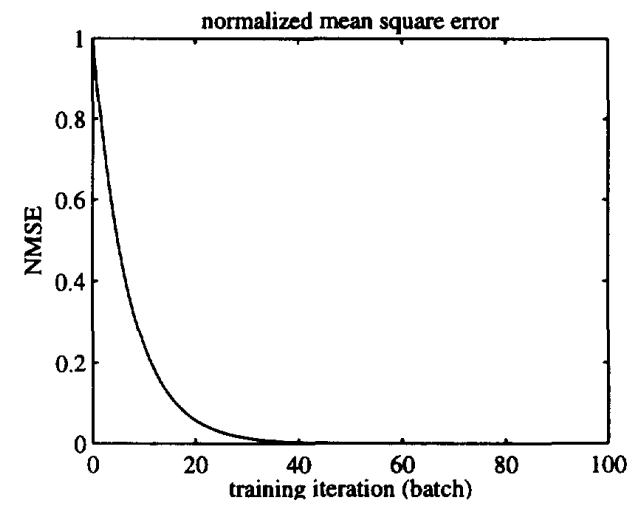

Figure 2: Case I: normalized mean square error of iteratively trained LAM coefficients and the closed form MACE filter for pre-whitened training patterns. The pre-whitening filter is computed over the average of the recognition class training patterns. 
The plot of figure 2 shows the mean square error between the effective space-domain coefficients of the LAM and the closed form MACE filter. As can be seen the error decreases in very few iterations to a negligible level.

\section{B. Case II}

In the second experiment the same regions. 0 through 2 were used as training patterns for the LAM, however the whitening filter was computed using the average spectrum of all numbered regions in the image of figure 1 . Both sets of filter coefficients were computed as before. As in case I the LAM was trained as in the space domain. Figure 3 shows the mean square error between the effective space-domain coefficients of the LAM and the closed form MACE filter. As in the first case the error decreases in a very few iterations to a negligible level.

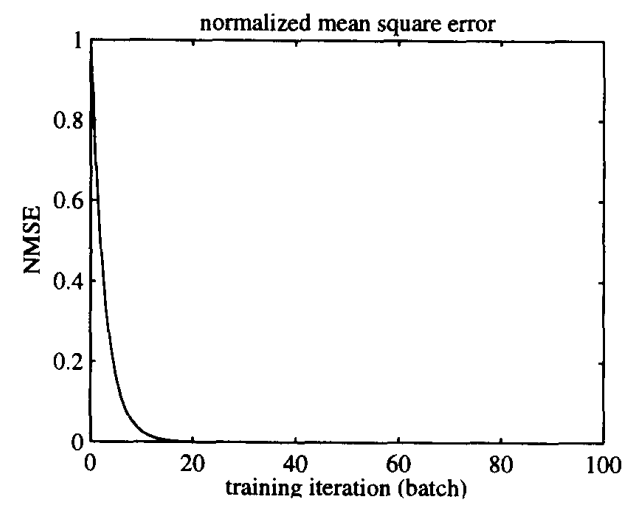

Figure 3: Case II: normalized mean square error of the LAM coefficients versus MACE filter coeffcients. In this case the pre-whitening filter is computed over the average of all numbered patterns.

\section{Conclusions}

It was shown that the MACE filter and the LAM trained with appropriately pre-whitened inputs are equivalent. The LAM formulation also offers the other advantages stated previously, that of iterative computation yielding a least squares solution even if the MACE filter formulation results in a singular matrix inversion. It also does not require as much memory storage as the computation is local and not averaged over all training patterns in one computational step.

The experimental results indicate that the convergence to the solution using the Widrow-Hoff rule is fairly quick. As stated initializing the weight vector to zero guarantees convergence to the minimum norm solution.

The LAM formulation also enables a computation of the capacity of the MACE filter as well as exten- sions to nonlinear processing which may improve the generalization properties.

\section{REFERENCES}

[Duda] Richard O. Duda and Peter E. Hart; Pattern Classification and Scene Analysis, John Wiley \& Sons; 1973.

[Widrow] Bernard Widrow and Samuel D. Stearns; Adaptive Signal Processing, Prentice-Hall, Inc.; 1985.

[Mahal] A. Mahalanobis, B.V.K. Vijaya Kumar, and D. Cassesent; Minimum average correlation energy filters, Appl. Opt. 26 no. 17, 3633-3640; 1987.

[Kohonen] Kohonen, Teuvo; Self-Organization and Associative Memory, 2nd ed.; Springer Series in Information Sciences, vol. 8; Springer-Verlag; 1988.

[Amit] Amit, Daniel J.; Modeling Brain Function: The World of Atractor Neural Networks, Cambridge University Press; 1989.

[Opp and Schaf] Oppenheim, Alan V., Ronald W. Schafer; Discrete Time Signal Processing, Prentice-Hall, Inc.; 1989.

[Ravi] Gopalan Ravichandran, and D. Cassesent; Minimum noise and correlation energy optical correlation filter, Appl. Opt. 31 no. 11, 1823-1833; 1992. 